\title{
Comparison of immunohistochemistry and polymerase chain reaction for detection of West Nile virus in naturally infected dead birds
}

\author{
Tejbir Sandhu ${ }^{1,2}$, Dalbinder Sidhu², Major Dhillon ${ }^{1}$ and Ying Fang ${ }^{3}$ \\ ${ }^{1}$ Northwest Mosquito and Vector Control District, 1966 Compton Ave, Corona, California 92881, USA \\ ${ }^{2}$ Department of Zoology, Punjabi University, Patiala 147002, India \\ ${ }^{3}$ Center for Vectorborne Diseases, Department of Pathology, Microbiology and Immunology, School of Veterinary \\ Medicine, University of California, Davis, California 95616, USA
}

\begin{abstract}
Introduction: Credible vector-borne disease surveillance programs, especially in developing countries with limited resources, must include diagnostic tests that are efficient, inexpensive and simple and safe to administer while maintaining high levels of sensitivity and specificity. Since immunohistochemistry (IHC) includes most of these features, its sensitivity, specificity, predictive positive value (PPV) and predictive negative value (PNV) for West Nile virus (WNv) screening were compared to those of the gold standard, RT-PCR testing of kidney tissue in dead birds.

Methodology: IHC and RT-PCR were performed for WNv antigen on 41 dead birds (belonging to five orders) collected from the northwest region of the Riverside County of California. Fixed tissue sections were screened by IHC using polyclonal antibodies, and frozen kidney tissues were tested with RT-PCR.

Results: Kidney screening with IHC showed sensitivity, specificity, PPV and NPV of $95.45 \%, 73.68 \%, 80.77 \%$ and $93.33 \%$, respectively. Based on WNv screening of kidney tissue, IHC and RT-PCR were in agreement with $95.45 \%$ (21/22) for positive dead birds and were in $100 \%$ (22/22) agreement when multi-organ screening by IHC was performed.

Conclusions: The present study showed that IHC is as equally effective as RT-PCR in screening for WNv in dead birds. Therefore, IHC can effectively serve as a competent screening technique for those disease surveillance agencies that lack expensive RT-PCR technology while promoting safer biohazardous conditions, except at the initial stage of tissue collection.
\end{abstract}

Key words: immunohistochemistry; RT-PCR; West Nile virus; vector-borne disease surveillance

J Infect Dev Ctries 2010; 4(9):587-589.

(Received 11 February 2010 - Accepted 04 June 2010)

Copyright () 2010 Sandhu et al. This is an open-access article distributed under the Creative Commons Attribution License, which permits unrestricted use, distribution, and reproduction in any medium, provided the original work is properly cited.

\section{Introduction}

In nature, birds act as a reservoir for disease as well as an amplifying host for the transmission of West Nile virus (WNv). For $\mathrm{WNv}$ disease surveillance, not only mosquitoes, but birds are an equally important component for determining the epidemiological ramifications of the disease. It is known that some species of dead birds (order: Passeriformes) are the earliest indicators of $\mathrm{WNv}$ activity [1]. Presently, in California, USA, it is a common practice to conduct $\mathrm{WNv}$ testing of dead birds with reverse transcriptase polymerase chain reaction (RT-PCR) by screening kidney tissue [2]. A PCR laboratory is expensive, requiring specialized equipment and technically trained personnel, which most developing countries have difficulty obtaining. Similar to PCR, immunohistochemistry (IHC) is also a powerful diagnostic technique for $\mathrm{WNv}$ screening.
Credible disease surveillance programs, especially in the developing countries with limited resources, must include diagnostic tests that are efficient, inexpensive, and simple and safe to administer while maintaining high levels of sensitivity and specificity. Since IHC includes most of these features, its sensitivity, specificity, predictive positive value (PPV) and predictive negative value (PNV) for $\mathrm{WNv}$ screening were compared to those of RT-PCR kidney screening in dead birds, especially those belonging to order Passeriformes which are primarily implicated as a reservoir for disease and amplification host for WNv. IHC can effectively serve as a diagnostic tool for those disease surveillance agencies that do not have the infrastructure to set up an expensive and comprehensive RT-PCR laboratory for $\mathrm{WNv}$ screening of dead birds. 
Table 1. Comparison between screening results for $\mathrm{WNv}$ using RT-PCR and IHC

\begin{tabular}{|l|l|l|l|}
\hline & Kidney tissue & IHC +ve & IHC -ve \\
\hline PCR & All birds & 21 & 1 \\
+ve & Order: Passeriformes & 20 & 1 \\
& American crows & 19 & 1 \\
\hline PCR & All birds & 5 & 14 \\
-ve & Order: Passeriformes & 5 & 9 \\
& American crows & 5 & 6 \\
\hline
\end{tabular}

Total birds: 41. Order Passeriformes: 35. American crows (Corvus brachyrhvnchos): 31

\section{Methodology}

IHC and RT-PCR were performed on 41 dead birds, which were assumed to be dead within 24 hours. The dead birds were collected from the northwest region of Riverside County, California. Dead bird sightings were reported by the public to either the state's toll-free hotline (i.e., 1-877-WNVBIRD, 1-877-968-2473) or directly to the Northwest Mosquito and Vector Control District (NWMVCD) office in Corona, California. Dead bird reporting calls received at the state hotline were procedurally forwarded to the NWMVCD office. Upon receiving calls, dead birds were immediately picked up by the district staff and were transported on ice to the NWMVCD laboratory for necropsies. All dead birds were found individually and not alongside other dead birds. The species of birds were identified using a field bird guide [3] and further confirmed by an ornithologist. The bird necropsies were conducted under a biological hood in a negatively pressurized room. Liver, kidney, spleen, heart, lungs, brain, pancreas, intestines, gonads, and optic tantrum were collected in $10 \%$ buffered formalin for producing histological sections. Since autolysis can obscure subtle lesions, tissues with minimal autolysis were chosen for microscopic evaluation. A quartered section of one kidney from each bird was collected and stored without any additive at $-80^{\circ} \mathrm{C}$ in cryogenic vials for screening with the singleplex RT-PCR Taqman assay (7900HT Fast RT-PCR system, Applied Biosystems by Life technologies, USA). After 24 hours of fixation in the formalin, these tissue samples were processed for histological sectioning. Paraffin tissue blocks were sectioned at $5 \mu \mathrm{m}$ thickness and mounted on positively charged glass slides. All tissue sections were screened for WNv using an immunoperoxidase method (Envision system: DAKO Corporation Carpinteria, CA), performed according to the manufacturer's instructions [4]. All immunostained slides were counterstained with hematoxylin. Because of the small size of the bird organs, multiple organs from the same bird were collectively screened on a single slide, thus not only reducing costs in labour and reagent usage, but also increasing the probability of detecting the $\mathrm{WNv}$ infection. Any carcass that was positive for $\mathrm{WNv}$ in at least one of the organs was designated infected. After staining, all the slides were examined under a light microscope. All positive tissue slides were digitally photographed for permanent record.

\section{Results}

A total of 41 dead birds belonging to five orders namely Passeriformes (35), Columbiformes (2), Strigiformes (2), Psittaciformes (1) and Falconiformes (1) were tested in this study. Out of the 35 Passeriform birds collected, 31 were American crows (Corvus brachyrhynchos). Gross examination showed subcutaneous hemorrhage on the skull in $32 \%$ of the $\mathrm{WNv}$ positive birds, thus indicating signs of trauma. Presence of stained $\mathrm{WNv}$ antigen was observed in brain, spleen, heart, kidney, liver, gonads, intestine, lung, pancreas and optic tectum. The spleen was the most frequently infected organ with $\mathrm{WNv}$, followed equally by the kidney and liver.

Based on kidney tissue testing of these 41 birds, IHC had a sensitivity of $95.45 \%$ and specificity of $73.68 \%$ with predictive positive value (PPV) of $80.77 \%$ and predictive negative value (PNV) of 93.33\% (Table 1). The relationship between RT-PCR and IHC for WNv-positive birds was $95.45 \%$ (21/22), and increased to $100 \%(22 / 22)$ when multiple organs from the same bird were screened with IHC. The sensitivity, specificity, PPV and PNV for birds of the order Passeriformes were 95.24\%, 64.29\%, 80\% and $90 \%$, respectively while American crows (the most sensitive bird to $\mathrm{WNv}$, showing the highest mortality rate) were $95 \%, 54.54 \%, 79.17 \%$ and $85.70 \%$, respectively (Table 2). Sample size for other orders of birds was too small to draw any statistically significant conclusion.

\section{Discussion}

Since our sample population of birds was found dead in the wild, the route of infection for $\mathrm{WNv}$ is presently unknown. The antigenic distribution of WNv among different organs of birds might depend upon their route of infection. The majority of these dead birds were American crows (order: Passeriformes). This order, i.e., American crows, house finches, house sparrows, blue jays, etc., are primarily implicated as the reservoir for disease and 
Table 2. Sensitivity, specificity, PPV and PNV of IHC with respect to screening of WNv

\begin{tabular}{|l|l|l|l|}
\hline Kidney tissue & Total birds $(\mathbf{n}=\mathbf{4 1})$ & Order: Passeriformes $(\mathbf{n}=\mathbf{3 5})$ & A crows $(\mathbf{n}=\mathbf{3 1})$ \\
\hline Sensitivity (\%) & 95.45 & 95.24 & 95.00 \\
\hline Specificity (\%) & 73.68 & 64.29 & 54.54 \\
\hline PPV (\%) & 80.77 & 80.00 & 79.17 \\
\hline PNV (\%) & 93.33 & 90.00 & 85.70 \\
\hline
\end{tabular}

amplification hosts for $\mathrm{WNv}$ [4], IHC screening can serve as a competent screening technique for those disease surveillance agencies that lack an RT-PCR facility. The present study showed that, compared to RT-PCR, IHC is less expensive but equally effective in screening $\mathrm{WNv}$ in dead birds. It is also less biohazardous to laboratory personnel except at the initial stage of tissue collection. Due to the small size of bird organs, multiple organs from the same bird were collectively screened on a single slide with the same amount of reagents and labour, thus increasing the probability of detecting $\mathrm{WNv}$ infection and eliminating additional work. For maximizing WNv detection in dead birds with IHC, it is highly recommended to screen multiple body organs (especially spleen, kidney, liver, intestine). IHC has another advantage of correlating the visual destruction of tissue architecture with the presence of stained $\mathrm{WNv}$ antigen, thus minimizing false positives. Additionally, formalin-fixed or paraffin embedded tissue samples can be archived, re-sectioned and restained for confirmation, and can also be used to screen for new or emerging pathogens in the future. As compared to virus isolation methodology, The IHC method is faster than viral isolation for the detection of WNv. However, IHC is still slower than RT-PCR testing. Rapid detection is necessary for quick implementation of vector control measures, limiting the usefulness of IHC.

\section{Acknowledgements}

We sincerely thank Gregory Williams for assisting in the identification of dead birds, Bryan Haynes for providing logistic support in collecting dead birds from the public, and Professor William Reisen, University of California, Davis, California, for allowing us to use his RT-PCR laboratory facility.

\section{References}

1. Eidson M, Komar N, Sorhage F, Nelson T, Talbot T, Mostashari F, McLean R (2001) Crow Deaths as a Sentinel Surveillance System for West Nile Virus in the Northeastern United States, 1999. Emerg Infec Dis 7: 615-620.

2. CDPH (California Department of Public Health). 2009. California Arbovirus Surveillance Bulletin. 44: 5.

3. Dunn JL and Alderfer J (2006) Field guide to the birds of North America, 5th edition. Washington DC: National Geographic Society. 481p.

4. Steele KE, Linn MJ, Schoepp RJ, Komar N, Geisbert TW, Manduca RM, Calle PP, Raphael BL, Clippinger TL, Larsen T, Smith J, Lanciotti RS, Panella NA, McNamara TS (2000) Pathology of fatal West Nile virus infections in native and exotic birds during the 1999 outbreak in New York City, New York. Vet Pathol 37: 208-224.

\section{Corresponding author}

Tejbir Sandhu

1966 Compton Ave

Corona, California 92882, USA

Tel: +1-951-340-9792; Fax: +1-951-340-2515

tsandhu@northwestmvcd.org

Conflict of interests: No conflict of interests is declared. 